\title{
Geometry and Physics of Null Infinity
}

\author{
Abhay Ashtekar* \\ Institute for Gravitation and the Cosmos 86 Physics Department, \\ Penn State, University Park, PA 16802, U.S.A.
}

\begin{abstract}
In asymptotically Minkowski space-times, one finds a surprisingly rich interplay between geometry and physics in both the classical and quantum regimes. On the mathematical side it involves null geometry, infinite dimensional groups, symplectic geometry on the space of gravitational connections and geometric quantization via Kähler structures. On the physical side, null infinity provides a natural home to study gravitational radiation and its structure leads to several interesting effects such as an infinite dimensional enlargement of the Poincaré group, geometrical expressions of energy and momentum carried by gravitational waves, emergence of non-trivial 'vacuum configurations' and an unforeseen interplay between infrared properties of the quantum gravitational field and the enlargement of the asymptotic symmetry group. The goal of this article is to present a succinct summary of this subtle and beautiful interplay.
\end{abstract}

\section{INTRODUCTION}

General relativity is widely regarded as the deepest realization of the rich interplay between geometry and physics known to date. The centerpiece of the theory is the fusion of the gravitational field with space-time geometry. Consequently, space-time geometry now becomes a dynamical entity and physics is encoded in its properties. The most profound predictions of the theory can be traced back to this interplay. It is because geometry is unleashed from its fixed, rigid structure that the universe can expand, we can have black holes and ripples of curvature can propagate across cosmological distances carrying away energy and momentum.

However, this very duality between geometry and gravity that makes it difficult to develop mathematical tools that are necessary to probe the general relativistic effects. An excellent example is provided by isolated gravitating systems. In the study of other fundamental interactions - such as electromagnetic - we have a background Minkowski space-time at our disposal, which is completely insensitive to the specific solutions we study. Using this 'inert', background geometry, then, it is easy to introduce physically motivated boundary conditions, such as the $1 / r$ fall-off on fields, and arrive at well-defined expressions of energy, momentum and angular momentum carried by electromagnetic waves. In general relativity, the metric which gives meaning to the 'fall-off conditions' is itself the dynamical variable on which we wish to impose the boundary conditions. This dual role creates unfamiliar layers of complexity. It took some 4 decades after the discovery of the theory to understand the boundary conditions at spatial infinity and to define the total energy, momentum [1] and angular momentum [2] of isolated systems [3]. It took another 2 decades to develop the

*Electronic address: ashtekar@gravity.psu.edu 
required geometric analysis to prove that, if the local energy density in matter is positive, then the total energy is also positive $[4,5]$.

Gravitational radiation adds yet another level of subtleties. Already in 1917, Einstein had isolated the radiative modes of the gravitational field in the linear approximation and derived the celebrated quadrupole formula. But subsequently, for several decades, there was a debate on whether this radiation was just an artifact of linearization. If one removes the anchor of a background Minkowski space-time geometry, can one still distinguish physical gravitational waves from coordinate effects in full, non-linear general relativity? It is interesting to note that at one stage Einstein himself thought one could not. He wrote: "Together with a young collaborator I arrived at the interesting result that gravitational waves do not exist, though they had been assumed to be a certainty to the first approximation. This shows that non-linear gravitational field equations tell us more or, rather, limit us more than we had believed up to now" (see, e.g., [6]).

It was only in the 1960s that Bondi, Sachs and their coworkers resolved the long standing confusion by constructing a framework in which the issue could be analyzed in an invariant fashion [7-9]. Specifically, because gravitational waves travel within light cones in general relativity, they constructed a systematic expansion of the metric as one moves away from the sources in null directions and studied asymptotics at null infinity, in contradistinction with the Arnowitt Deser Misner (ADM) framework which focuses on spatial infinity. This construction was cast in a more convenient form through conformal techniques by Penrose [10] where null infinity is represented as the boundary, $\mathcal{I}$, of the physical space-time in its conformal completion. As this article will show, Penrose's $\mathcal{I}$-framework has rich geometry containing important physics.

Starting from the late 1970s, the geometric analysis community has studied the ADM framework and, more generally, the global elliptic problems associated with the initial value formulation of Einstein's equations in great detail (see, e.g., [11] for a review). Similar investigations of the hyperbolic problems related to $\mathcal{I}$ were undertaken later, using Einstein's equations on the physical metric [12], as well as on the conformally rescaled metric which is well-behaved at $\mathcal{I}$ [13]. Over the years, there has been considerable progress [14, 16], and this area is likely to witness significant advances over the next decade (for the current status, see, e.g., [17]).

On the physical side, the framework provides the conceptual foundation for a surprisingly large portion of gravitational science. In particular, it provides the basis for analytical approximation schemes as well as numerical simulations of the emission of gravitational waves in gravitational collapse and binary coalescence. It lies at the heart of the definition of black holes and is therefore important for all of the related mathematical physics. (See, e.g., [18-20] for reviews.) Finally, in quantum gravity, it provides a natural arena for the $S$-matrix theory and is required in the formulation of conceptual issues such as that of information loss during black hole evaporation and the role of the CPT symmetry in quantum gravity (see, e.g., [21-24]). On the physical side, interest in the structure of $\mathcal{I}$ has increased recently through more detailed analyses of, e.g., the 'memory' effect [25-27] in the classical theory, and a resurgence in the investigations of the representations of the Bondi Metzner Sachs (BMS) group [28, 29], scattering amplitudes [30, 31] and the subtle infrared issues [32-34] on the quantum side.

Therefore, an overview of geometry and physics of $\mathcal{I}$ seems appropriate at this juncture. Furthermore, structure of $\mathcal{I}$ serves as a striking illustration of the power of the interplay between these two disciplines that general relativity embodies, and this is a jubilee volume 
celebrating the 100th anniversary of Einstein's theory. However, because space is limited, this will be a broad overview without technical details or proofs. But a special attempt has been made to address both mathematics and physics communities.

In section II we recall the basic structure of $\mathcal{I}$, including the enlargement of the Poincaré group to the infinite dimensional BMS group. In section III we discuss the interplay between geometry and physics that dominates the description of radiative modes of the gravitational field in full general relativity. Finally, in section IV we discuss some of the quantum issues. Our conventions are as follows. Space-time will be assumed to be 4-dimensional and its metric will have signature,,,-+++ . Physical fields will carry hats while those which are well defined on the conformal completion will be unhatted. We will use Penrose's abstract index notation [35] where the indices serve as markers to denote the type of tensor field being considered and do not refer to a chart. The curvature tensors (in the completion) are defined via: $2 \nabla_{[a} \nabla_{b]} k_{c}=R_{a b c}{ }^{d} k_{d}, R_{a c}=R_{a b c}{ }^{b}$ and $R=R_{a b} g^{a b}$. For simplicity of presentation we will assume that all fields are $C^{\infty}$. However, the main results discussed here hold for the weaker smoothness at $\mathcal{I}$ assured by the Christodoulou-Klainnermann analysis [12, 14].

\section{THE BONDI-PENROSE FRAMEWORK}

This section is divided into two parts. In the first we introduce the relevant definitions of asymptotic flatness and summarize their immediate consequences. In the second we discuss the structure of the asymptotic symmetry group.

\section{A. Basic structure}

Study of null infinity is based on two basic definitions [36, 37]. The first is weaker and suffices for the analysis of gravitational radiation at $\mathcal{I}$, while the second and stronger becomes necessary to address global issues. In what follows, $\mathcal{I}$ will stand either for the future or the past boundaries $\mathcal{I}^{ \pm}$of space-time. For the gravitational radiation theory and black holes one is primarily interested in future null infinity, $\mathcal{I}^{+}$.

Definition 1: A space-time $\left(\hat{M}, \hat{g}_{a b}\right)$ will be said to be asymptotically flat at null infinity if there exists a manifold $M$ with boundary $\mathcal{I}$ equipped with a metric $g_{a b}$ and a diffeomorphism from $\hat{M}$ onto $M \backslash \mathcal{I}$ (with which we identify $\hat{M}$ and $M \backslash \mathcal{I}$ ) such that:

i) there exists a smooth function $\Omega$ on $M$ with $g_{a b}=\Omega^{2} \hat{g}_{a b}$ on $\hat{M} ; \Omega=0$ on $\mathcal{I}$; and $n_{a}:=\nabla_{a} \Omega$ is nowhere vanishing on $\mathcal{I}$;

ii) $\mathcal{I}$ is topologically $\mathbb{S}^{2} \times \mathbb{R}$; and,

iii) $\hat{g}_{a b}$ satisfies Einstein's equations $\hat{R}_{a b}-\frac{1}{2} \hat{R} \hat{g}_{a b}=8 \pi G \hat{T}_{a b}$, where $\Omega^{-2} \hat{T}_{a b}$ has a smooth limit to $\mathcal{I}$.

The first condition ensures that $\left(M, g_{a b}\right)$ is a conformal completion of the physical spacetime $\left(\hat{M}, \hat{g}_{a b}\right)$ in which the boundary $\mathcal{I}$ is at infinity with respect to the physical metric $\hat{g}_{a b}$. The condition $\nabla_{a} \Omega \neq 0$ on $\mathcal{I}$ ensures that $\Omega$ can be used as a coordinate on $M$; we can perform Taylor expansions in $\Omega$ to capture the degree of fall-off of physical fields. In terms of the physical space-time $\left(\hat{M}, \hat{g}_{a b}\right)$, it ensures that $\Omega$ 'falls-off as $1 / r$ ', i.e., has the same asymptotic behavior as in the standard conformal completion of Minkowski spacetime. The topological restriction captures the idea that one can move away from the isolated 
system along null rays in any angular direction. The last condition ensures that the matter fields fall-off appropriately in the physical space-time $\left(\hat{M}, \hat{g}_{a b}\right)$. The specific fall-off of $\hat{T}_{a b}$ is motivated by the analysis of test fields in Minkowski and Schwarzschild space-times.

These conditions immediately imply that $\mathcal{I}$ is necessarily a null, 3 -dimensional manifold. Therefore $n^{a}=\nabla^{a} \Omega$ is null and $\mathcal{I}$ is ruled by the the integral curves of $n^{a}$, called the generators. The space $S$ of generators is topologically $\mathbb{S}^{2}$. The pull-back $q_{a b}:=g_{a b}$ of the conformally rescaled metric to $\mathcal{I}$ has signature $0,+,+$ and is the lift to $\mathcal{I}$ of a positive definite metric $\bar{q}_{a b}$ on $S$.

Next, note that there is freedom to perform conformal rescalings: If $\Omega$ is a permissible conformal factor for a physical space-time $\left(\hat{M}, \hat{g}_{a b}\right)$, so is $\Omega^{\prime}=\omega \Omega$ where $\omega$ is smooth on $M$ and nowhere vanishing on $\mathcal{I}$. Using this freedom, one can always choose a conformal completion such that $\nabla_{a} n^{a}$ vanishes on $\mathcal{I}$. We will always work with such a divergence-free conformal frame. The fall-off condition iii) in Definition 1 implies that in these frames, a stronger condition is automatically satisfied:

$$
\nabla_{a} n_{b}:=\nabla_{a} \nabla_{b} \Omega \hat{=} 0
$$

Here and throughout the rest of the paper, whenever there may be an ambiguity on whether a given equality holds on all of $M$ or just on $\mathcal{I}$, we will use the symbol $\hat{=}$ to denote equality restricted to $\mathcal{I}$. The remaining conformal freedom is given by $\Omega^{\prime}=\omega \Omega$ where $\mathcal{L}_{n} \omega \hat{=} 0$. Eq. (2.1) implies that the torsion-free derivative operator $\nabla$ compatible with $g_{a b}$ induces a torsion-free derivative operator $D$ defined intrinsically on the 3 -manifold $\mathcal{I}$, satisfying

$$
D_{a} q_{b c}=0, \quad \text { and } \quad D_{a} n^{b}=0 .
$$

Finally, Definition 1 implies that the Weyl tensor $C_{a b c}{ }^{d}$ of $g_{a b}$ vanishes on $\mathcal{I}$. Therefore, the tensor field

$$
K_{a b c}{ }^{d}:=\Omega^{-1} C_{a b c}{ }^{d}
$$

has a smooth limit to $\mathcal{I}$ and is called the leading order Weyl tensor at $\mathcal{I}{ }^{1}$

Fix any two divergence-free conformal frames $\Omega$ and $\Omega^{\prime}$. Then, since the relative conformal factor satisfies $\mathcal{L}_{n} \omega \hat{=} 0$, the vector field $n^{a}$ is complete if and only if $n^{\prime a}$ is complete. Definition 1 does not demand completeness of $\mathcal{I}$ in the $\mathbb{R}$ direction. The second definition does, i.e., it asks that $\mathcal{I}$ have the same global structure as it has in the standard completion of Minkowski space-time.

Definition 2: An asymptotically flat space-time is said to be asymptotically Minkowski if $\mathcal{I}$ is complete in any divergence-free conformal frame.

The second notion is important for black holes [36]. Recall that a space-time $\left(\hat{M}, \hat{g}_{a b}\right)$ admits a black hole if the past $J^{-}\left(\mathcal{I}^{+}\right)$of the future null infinity is a proper subset of $\hat{M}$. Therefore, without the completeness requirement, one could carry out a conformal

\footnotetext{
${ }^{1}$ Given a null frame à la Newman and Penrose [10,35], the five complex functions $\Psi_{4}^{o}, \ldots \Psi_{0}^{o}$ capture the 10 components of $K_{a b c}{ }^{d}$ at $\mathcal{I}$. The 'peeling' properties of the $5 \mathrm{NP}$ scalars is a straightforward consequence of smoothness of $K_{a b c}{ }^{d}$ and the relation between the physical null tetrad defined by $\hat{g}_{a b}$ and the conformally rescaled one defined by $g_{a b}$. If differentiability at $\mathcal{I}$ is weaker as in [12] and [14], one has peeling only for some of the NP scalars.
} 
completion of Minkowski space-time which attaches to it just a "part of the $\mathcal{I}^{+}$of the standard completion' and conclude that it admits a black hole region! Completeness is also necessary in the discussion of the BMS group (as opposed to the BMS Lie algebra). However, there are examples (e.g. boost-symmetric space-times) in which this requirement is not met but one can meaningfully discuss gravitational radiation [37].

\section{B. The BMS Group}

The asymptotic symmetry group $\mathfrak{B}$ is the quotient $\operatorname{Diff}_{\infty}(M) / \operatorname{Diff}_{\infty}^{o}(M)$ of the group $\operatorname{Diff}_{\infty}(M)$ of diffeomorphisms on the physical space-time $\left(\hat{M}, \hat{g}_{a b}\right)$ that preserve the boundary conditions (imposed in Definition 2) by its subgroup Diffo ${ }_{\infty}^{o}(M)$ of diffeomorphisms that are asymptotically identity. This is the BMS group. At the infinitesimal level, elements of the Lie algebra $\mathfrak{b}$ of $\mathfrak{B}$ can be naturally represented by vector fields $\xi^{a}$ on $\mathcal{I}$, motions along which preserve the universal structure, i.e., the structure that is shared by all space-times satisfying Definition 2.

From our summary in the last sub-section, it follows that $\mathcal{I}$ is endowed with the following universal structure. It is topologically $\mathbb{S}^{2} \times \mathbb{R}$, and equipped with pairs of fields $\left(q_{a b}, n^{a}\right)$ such that: i) $q_{a b}$ is a degenerate metric of signature $0,+,+$ with $q_{a b} n^{b}=0$ and $\mathcal{L}_{n} q_{a b}=0$; ii) $n^{a}$ is complete; and, iii) any two pairs $\left(q_{a b}, n^{a}\right)$ and $\left(q_{a b}^{\prime}, n^{\prime a}\right)$ in the collection are related by a conformal rescaling,

$$
q_{a b}^{\prime}=\omega^{2} q_{a b} \text { and } \quad n^{\prime a}=\omega^{-1} n^{a},
$$

where $\mathcal{L}_{n} \omega=0$. Note that, because 2 -spheres carry a unique conformal structure, every $q_{a b}$ in this collection is conformal to a unit 2-sphere metric.

It then follows that $\mathfrak{B}$ can be characterized intrinsically in terms of $\mathcal{I}$ as the subgroup of diffeomorphisms of $\mathcal{I}$ that preserves this universal structure. It is simplest to explore its structure by working at the infinitesimal level. A vector field $\xi^{a}$ on $\mathcal{I}$ will preserve the universal structure if and only if:

$$
\mathcal{L}_{\xi} q_{a b}=2 \alpha q_{a b}, \quad \text { and } \quad \mathcal{L}_{\xi} n^{a}=-\alpha n^{a}
$$

for some function $\alpha$ on $\mathcal{I}$ satisfying $\mathcal{L}_{n} \alpha=0$. In particular, the vector fields $\xi^{a}=f n^{a}$ with $\mathcal{L}_{n} f=0$ satisfy this condition. Furthermore, the subspace $\mathfrak{s}$ they form in the Lie algebra $\mathfrak{b}$ of $\mathfrak{B}$ is a Lie ideal in the sense that $\left[\xi, f n^{a}\right] \in \mathfrak{s}$ for all $\xi^{a} \in \mathfrak{b}$ and all $f n^{a} \in \mathfrak{s}$. This is the Lie ideal of BMS supertranslations.

Next, note that the condition $\mathcal{L}_{\xi} n^{a}=\alpha n^{a}$ also implies that every BMS vector field $\xi^{a}$ can be projected to a vector field $\bar{\xi}^{a}$ on the 2 -sphere $S$ of generators of $\mathcal{I}$ which then characterizes the element of the quotient $\mathfrak{b} / \mathfrak{s}$ it naturally defines. Furthermore, the condition that the space of pairs $\left(q_{a b}, n^{a}\right)$ be preserved by the BMS action implies that $\bar{\xi}^{a}$ is a conformal Killing field on the space $S$ of generators of $\mathcal{I}$, equipped with metrics $\bar{q}_{a b}$. Since every $\bar{q}_{a b}$ is conformal to a round 2-sphere metric, it follows that the quotient $\mathfrak{b} / s$ is just the Lie algebra of conformal isometries of a round 2-sphere. But this is just the Lie algebra of the Lorentz group in 4 space-time dimensions. Returning to finite diffeomorphisms, we conclude that $\mathfrak{B}$ is the semi-direct product, $\mathfrak{B}=\mathcal{S} \ltimes \mathcal{L}$, of the group $\mathcal{S}$ of supertranslations with the Lorentz group $\mathcal{L}$. Thus, although $\mathfrak{B}$ is infinite dimensional, its structure mimics that of the Poincaré group, the 4-dimensional Abelian group of translations being replaced by that by the infinite dimensional Abelian group $\mathcal{S}$ of supertranslations. Because each supertranslation 
is represented by a vector field of the type $f n^{a}$ on $\mathcal{I}$ where $f$ is the pull-back to $\mathcal{I}$ of a function $\bar{f}$ on the 2 -sphere $S$ of generators, supertranslations can be regarded as 'angle dependent translations' in the physical space-time. While the Poincaré group admits a 4-parameter family of Lorentz groups, each labeled by a point in Minkowski space-time, the BMS group has an infinite number of Lorentz subgroups, each labeled by a 2 -sphere cross section of $\mathcal{I}$ (i.e., a lift of $S$ to $\mathcal{I}$ ).

However, because the space of generators of $\mathcal{I}$ is topologically $\mathbb{S}^{2}$, one can show that $\mathfrak{B}$ also admits a unique normal, Abelian subgroup $\mathcal{T}$ [8]. This is a subgroup of $\mathcal{S}$ and in Minkowski space-time it coincides with the group of space-time translations. Because there is a canonical translation subgroup $\mathcal{T}$, the notion of 4 -momentum continues to be welldefined for gravitational waves, and also for matter waves in the presence of gravitational radiation. But the familiar notion of angular-momentum we use in Minkowski space-time now acquires an infinite dimensional, supertranslation ambiguity. Thus, the enlargement from the Poincaré to the infinite dimensional BMS group has direct physical consequences but they are subtle. We will see in section III that the enlargement is directly related to the presence of gravitational waves.

Finally, as one would expect, every Killing vector $K^{a}$ of the physical space-time satisfying Definition 1 extends naturally to a BMS vector field on $\mathcal{I}$. Furthermore, if the leading order Weyl curvature is such that $K_{a b c d} n^{d}$ is not identically zero at $\mathcal{I}$, then the isometry group is at most 4-dimensional, and a subgroup of the isometry group of the Schwarzschild space-time. If the extension of $K^{a}$ is a supertranslation on $\mathcal{I}$, then it is necessarily a time translation in $\mathcal{T}[37]$.

Remark: It is sometimes convenient to further restrict the conformal freedom at $\mathcal{I}$ by demanding that the metric $\bar{q}_{a b}$ on the space $S$ of generators be a unit 2-sphere metric. This is always possible and these conformal frames are called Bondi frames. In a Bondi frame, a BMS supertranslation $\xi^{a}=f n^{a}$ is a translation if and only if

$$
D_{a} D_{b} f \propto q_{a b}
$$

This is equivalent to asking that $f$ be a linear combination of the first four spherical harmonics, $f=f_{0} Y_{0,0}(\theta, \phi)+f_{m} Y_{1, m}(\theta, \phi)$ for some real constants $f_{0}, f_{m}$ (with $\left.m=-1,0,1\right)$. However, restricting oneself to Bondi frames can also make certain conceptual issues more difficult to analyze, especially in the definition of the 'BMS charges' — which generalize the notions of energy-momentum and angular momentum in view of the enlargement of the Poincaré group to the BMS - and the fluxes of BMS momenta across $\mathcal{I}$ [38].

\section{RADIATIVE MODES IN EXACT GENERAL RELATIVITY}

This section is divided into three parts. In the first, we explore higher (asymptotic) order geometrical structures that are not universal and encode physical information of the given space-time; in the second, we introduce the notion of 'vacuum configurations' following gauge theories, and in the third we discuss the symplectic geometry of radiative modes and momentum maps defined by the BMS symmetries. 


\section{A. Further geometrical structures at $\mathcal{I}$}

The universal structure of $\mathcal{I}$ is common to all asymptotically Minkowski space-times. Therefore we will regard the $\mathbb{S}^{2} \times \mathbb{R}$ topology and the collection of pairs $\left(q_{a b}, n^{a}\right)$ as the zeroth order structure of $\mathcal{I}$. The first order structure is the connection $D$ defined intrinsically on $\mathcal{I}$ in any given conformal completion, induced by the torsion-free connection $\nabla$ compatible with $g_{a b}$. As we will see, it encodes the 'radiative information' in the physical space-time $\left(\hat{M}, \hat{g}_{a b}\right)$ and therefore varies from one space-time to another. In particular, Eqs. (2.2) do not determine $D$ uniquely because $q_{a b}$ is degenerate.

It is convenient to first fix a conformal frame $\left(q_{a b}, n^{a}\right)$ on $\mathcal{I}$, develop the framework, and then study what happens under conformal rescalings $g_{a b} \rightarrow g_{a b}^{\prime}=\omega^{2} g_{a b}$. However, even when $\omega=1$ on $\mathcal{I}$ so that $q_{a b}$ and $n^{a}$ are unchanged, $D$ transforms via $D_{a}^{\prime} k_{b}=D_{a} k_{b}+f\left(n^{m} k_{m}\right) q_{a b}$, where $k_{b}$ is an arbitrary 1 -form on $\mathcal{I}$ and $f$ is defined via $\nabla^{a} \omega \hat{=} f n^{a}$. Therefore we are led to introduce an equivalence relation:

$$
D \approx D^{\prime} \quad \text { iff } \quad\left(D_{a}^{\prime}-D_{a}\right) k_{b}=\left(f n^{c} k_{c}\right) q_{a b}
$$

where $f$ is an arbitrary function on $\mathcal{I}$. We will denote the equivalence class by $\{D\}$. Eqs. (2.2) now imply that any two connections in a given conformal frame $\left(q_{a b}, n^{a}\right)$ are related by $\left(D_{a}^{\prime}-D_{a}\right) k_{b}=\Sigma_{a b} n^{c} k_{c}$ where $\Sigma_{[a b]}=0$ and $\Sigma_{a b} n^{b}=0$. Therefore the difference $\left\{D^{\prime}\right\}-\{D\}$ between the equivalence classes of connections is completely characterized by the trace-free part

$$
\sigma_{a b}=\Sigma_{a b}-\frac{1}{2} \Sigma_{c d} q^{c d} q_{a b}
$$

of $\Sigma_{a b}$ (where $\Sigma_{c d} q^{c d}$ is well defined because $\Sigma_{a b} n^{b}=0$ ). The space $\boldsymbol{\Gamma}$ of equivalence classes $\{D\}$ is an affine space. Hence by choosing any $\{D\}_{0}$ as origin, any $\{D\}$ can be uniquely labeled by a symmetric, trace-free tensor field $\sigma_{a b}$ on $\mathcal{I}$ which is transverse, i.e., satisfies $\sigma_{a b} n^{b}=0$. Physically, the two independent components of $\sigma_{a b}$ can be regarded as representing the two radiative degrees of freedom of the gravitational field in exact general relativity [39]. It is striking that, in spite of the complicated non-linearities of general relativity, the structure of the radiative modes is so simple at $\mathcal{I}$. In a characteristic initial value problem, $\{D\}$ represents the part of the initial data that is freely specifiable on 3 -dimensional $\mathcal{I}$ (in addition to other quantities that have to be specified on a transverse null surface $\mathcal{N}$ and the 2- sphere at which $\mathcal{N}$ intersects $\mathcal{I}$ ) [40].

Since $\mathcal{I}$ is 3 -dimensional, the curvature tensor $R_{a b c}{ }^{d}$ of any $D$ is completely determined by a second rank tensor $S_{a}{ }^{b}$ :

$$
R_{a b c}{ }^{d}=q_{c[a} S_{b]}^{d}+S_{c[a} \delta_{b]}^{d},
$$

where $S_{a b}=S_{a}{ }^{c} q_{b c}$. Because of Eq. (2.2), the field $S_{a}{ }^{b}$ on $\mathcal{I}$ satisfies $S_{[a b]}=0, S_{a b} q^{a b}=\bar{R}$ and $S_{a}{ }^{b} n^{a} \propto n^{b}$, where $\bar{R}$ is the pull-back to $\mathcal{I}$ of the scalar curvature of $\bar{q}_{a b}$ on the 2sphere of generators $S$. One would expect $R_{a b c}{ }^{d}$ to carry physically important information about gravitational waves in the given space-time. This is indeed the case modulo a small complication. Let us first consider $S_{a b}$. The complication is that if we change the conformal frame, the curvature tensor transforms in a complicated manner. Thus, part of this curvature is 'gauge' in the sense that it does not carry any physical information. If $q_{a b}^{\prime}=\omega^{2} q_{a b}, n^{\prime a}=$ $\omega^{-1} n^{a}$ (with $\omega$ not necessarily equal to 1 at $\mathcal{I}$ ) we have:

$$
\begin{aligned}
D^{\prime}{ }_{a} k_{b} & \hat{=} D_{a} k_{b}-2 \omega^{-1} k_{(a} D_{b)} \omega+\omega^{-1}\left(\nabla^{m} \omega\right) k_{m} q_{a b} \\
S_{a b}^{\prime} & \hat{=} S_{a b}-2 \omega^{-1} D_{a} D_{b} \omega+4 \omega^{-2} D_{a} \omega D_{b} \omega-\omega^{-2}\left(q^{m n} D_{m} \omega D_{n} \omega\right) q_{a b} .
\end{aligned}
$$


$\left(q^{m n} D_{m} \omega D_{n} \omega\right.$ is well-defined because $\left.\mathcal{L}_{n} \omega=0\right)$. The task is to extract conformally invariant information from this rather complicated transformation property. This can be done in an elegant manner thanks to a result due to Geroch [41]: In any conformal frame $\left(q_{a b}, n^{a}\right)$, there is a unique 'kinematical' tensor field $\rho_{a b}$ on $\mathcal{I}$ satisfying:

$$
\rho_{[a b]}=0 ; \quad \rho_{a b} n^{b}=0 ; \quad \rho_{a b} q^{a b}=\bar{R} \text { and } D_{[a} \rho_{b] c}=0 .
$$

One can show that under conformal rescalings, the transformation property of $\rho_{a b}$ mimics that of $S_{a b}$ so that

$$
N_{a b}:=S_{a b}-\rho_{a b}
$$

is conformally invariant. Thus, the role of $\rho_{a b}$ is simply to remove from $S_{a b}$ the gaugedependent part. $N_{a b}$ is called the Bondi news tensor and represents the 'second order' structure at $\mathcal{I}$, constructed from the derivative of the first order structure encoded in $\{D\}$.

However, it is $S_{a}{ }^{b}$ rather than $S_{a b}$ that has the full information of the curvature of $\{D\}$. (One cannot reconstruct $S_{a}{ }^{b}$ from $S_{a b}$ because $q_{a b}$ is degenerate.) Can we also extract the gauge-independent part from full $S_{a}{ }^{b}$ ? This is indeed possible using the field $K^{a b c d}$ of Eq. (2.3) that captures the leading order, asymptotic space-time curvature. (Here the indices are of course raised using $\left.g^{a b}\right)$. The 'magnetic part'

$$
{ }^{\star} K^{a c}: \hat{=}{ }^{\star} K^{a b c d} n_{b} n_{d}
$$

of this asymptotic curvature is tangential to $\mathcal{I}$. It turns out that this part is related to $S_{a}{ }^{b}$ via

$$
{ }^{\star} K^{a c}=2 \epsilon^{m n a} D_{m} S_{n}{ }^{c},
$$

where $\epsilon^{a b c}=\epsilon^{a b c d} n_{d}$ is the intrinsic volume 3 -form on $\mathcal{I}$. Since ${ }^{\star} K^{a c}$ involves derivatives of $S_{a}{ }^{b}$, it represents the third order structure at $\mathcal{I}$. By definition it is also symmetric and trace-free. ${ }^{2}$ Under conformal rescalings, ${ }^{\star} K^{a b}$ transforms covariantly:

$$
{ }^{\star} K^{\prime a b}=\omega^{-5 \star} K^{a b}
$$

Finally one can show locally, i.e., without having to refer to completeness of $\mathcal{I}$, that if ${ }^{\star} K^{a b}=0$ then $N_{a b}=0[42] .{ }^{\star} K^{a b}$ captures the full gauge covariant information in the radiative mode $\{D\}$, in the same sense that the (Lie algebra-valued) curvature 2-form $F_{a b}$ captures the full gauge covariant local information in a non-Abelian connection 1-form $A_{a}$.

\section{B. Vacuum configurations and the Poincaré reduction}

If ${ }^{\star} K^{a b}=0$, the equivalence class $\{D\}$ of connections is said to be trivial. It is clear from Eq. (3.10) that this is a conformally invariant notion. In what follows, for brevity, we will drop the words 'equivalence class' and refer to $\{D\}$ simply as a connection. Following the terminology used in gauge theories in which the dynamical variable is again a connection,

\footnotetext{
${ }^{2}$ In a Newman-Penrose null tetrad [10,35], components of ${ }^{\star} K^{a c}$ are given by $\Psi_{4}^{o}, \Psi_{3}^{o}, \operatorname{Im} \Psi_{2}^{o}$. Note that the components do not include $\operatorname{Re} \Psi_{2}^{o}$, which represents the 'longitudinal mode' of the gravitational field and determines the mass in stationary space-times. For detailed derivations of the results quoted in this sub-section, see $[23,39]$.
} 
trivial connections are referred to as 'vacuum configurations', and denoted by $\{\stackrel{D}{D}\}$. If a physical space-time $\left(\hat{M}, \hat{g}_{a b}\right)$ is such that the induced connection on $\mathcal{I}$ is trivial, then it does not contain gravitational waves. In particular, as we will see below, there is no flux of energy or momentum or angular momentum at $\mathcal{I}$. All asymptotically flat, stationary space-times induce a trivial connection on $\mathcal{I}$. More generally, in physics one is interested only in those space-times in which gravitational radiation falls-off sufficiently fast in the distant past and in the distant future. Thus, all configurations $\{D\}$ of physical interest tend to vacuum configurations $\left\{\stackrel{\circ}{ }^{\mp}\right\}$ as we move to spatial infinity $i^{o}$ and future time-like infinity $i^{+}$along $\mathcal{I}^{+}$.

Let $\stackrel{\circ}{\Gamma}$ denote the subspace of $\boldsymbol{\Gamma}$ spanned by vacuum configurations. We will now explore the structure of $\stackrel{\Gamma}{\Gamma}$ and the action of the BMS group $\mathfrak{B}$ on it following [39]. As we noted in section III, the difference $\{D\}^{\prime}-\{D\}$ between any two connections in $\boldsymbol{\Gamma}$ is characterized by a trace-free symmetric tensor field $\sigma_{a b}$ that it transverse to $n^{a}$. One can show that if $\{D\}^{\prime}$ and $\left\{D^{\circ}\right\}$ are both in $\stackrel{\circ}{\Gamma}$ the difference has the following form in any Bondi conformal frame:

$$
\sigma_{a b}=D_{a} D_{b} s-\frac{1}{2}\left(q^{c d} D_{c} D_{d} s\right) q_{a b} \quad \text { for some function } s \text { on } \mathcal{I} \text { with } \quad \mathcal{L}_{n} s=0 .
$$

Since we are in a Bondi frame where $\bar{q}_{a b}$ is the unit 2 -sphere metric, the right side vanishes if and only if $s$ is the lift to $\mathcal{I}$ of $\bar{s}$ on $S$ of the form $\bar{s}=s_{0} Y_{0,0}+s_{m} Y_{1, m}, m=-1,0,1$, where $s_{0}$ and $s_{m}$ are constants. Thus, there are 'as many' vacuum configurations as there are elements of $\mathcal{F} / \mathcal{F}_{0,1}$ of the quotient of the space $\mathcal{F}$ of functions on $S$ by the 4-dimensional space $\mathcal{F}_{0,1}$ spanned by the first 4 spherical harmonics. Next, recall that a supertranslation is represented by a BMS vector field $\xi^{a}$ of the form $\xi^{a}=f n^{a}$ with $\mathcal{L}_{n} f=0$. Under the 1-parameter family of diffeomorphisms $d(\lambda)$ it generates, a connection $\{D\}$ is mapped to $\{D\}(\lambda)$. The difference $\{D\}(\lambda)-\{D\}$ is characterized by a 1-parameter family of tensor fields $\sigma_{a b}(\lambda)$ which, in a Bondi frame, has the form

$$
\sigma_{a b}(\lambda)=\lambda\left(D_{a} D_{b} f-\frac{1}{2}\left(q^{c d} D_{c} D_{d} f\right) q_{a b}\right)
$$

Eqs. (3.11) and (3.12) imply that:

(i) The action of supertranslations preserves the subspace $\boldsymbol{\Gamma}^{\circ}$ of $\boldsymbol{\Gamma}$ and the action is transitive;

(ii) A vacuum configuration $\{\stackrel{\circ}{D}\}$ is left invariant under this action if and only if $\xi^{a}$ is a BMS translation and BMS translations leave each vacuum configuration invariant.

Thus, the quotient $\mathcal{S} / \mathcal{T}$ of the group $\mathcal{S}$ of BMS supertranslations by its 4-dimensional normal subgroup $\mathcal{T}$ of translations acts simply and transitively on the space $\stackrel{\circ}{\Gamma}$. Note however that while this implies that $\mathcal{S} / \mathcal{T}$ is isomorphic to $\Gamma^{\circ}$, there is no natural isomorphism between them.

The discussion of section II B implies that the BMS group $\mathfrak{B}$ admits 'as many' Poincaré subgroups $\mathcal{P}$ as there are elements of $\mathcal{S} / \mathcal{T}$. More precisely, the group $\mathcal{S} / \mathcal{T}$ acts simply and transitively on the space $\boldsymbol{\Pi}$ of Poincaré subgroups $\mathcal{P}$ of $\mathfrak{B}$. Therefore it is natural to ask if there is a natural isomorphism between the two representation spaces, $\Gamma^{\circ}$ and $\Pi$, on both of which $\mathcal{S} / \mathcal{T}$ acts simply and transitively. The answer turns out to be in the affirmative.

Let us fix an element $\{\stackrel{\circ}{D}\}$ of $\stackrel{\circ}{\Gamma}$ and ask for the subgroup of the BMS group that leaves it invariant. A detailed analysis shows that it is precisely a Poincaré subgroup $\mathcal{P}_{\left\{D^{0}\right\}}$ of $\mathfrak{B}$ [39] (see also [43]). Furthermore, this map from $\stackrel{\circ}{\Gamma}$ to $\Pi$ is an isomorphism. Thus, the enlargement of the Poincaré to the BMS group at null infinity is directly related to the 
'vacuum degeneracy', i.e., to the fact that there are distinct connections $\{\stackrel{\circ}{D}\}$ which have trivial curvature in the sense that ${ }^{\star} K^{a b}$ they define vanishes identically (and therefore their Bondi news also vanishes). There is a precise sense in which this structure is analogous to that encountered in non-Abelian gauge theories in Minkowski space-time. However, there are also some key differences. Similarities and differences are both spelled out in [39].

From a gravitational physics perspective, the situation can be summarized as follows. As we already noted above, physically, one is interested in space-times which induce connections $\{D\}$ on $\mathcal{I}$ which asymptotically tend to vacuum configurations $\left\{\stackrel{\circ}{D}^{\mp}\right\}$ as we move along $\mathcal{I}^{+}$to the past towards $i^{o}$ and to the future towards $i^{+}$. For physically reasonable sources, generically $\left\{\stackrel{\circ}{D}^{\mp}\right\}$ are distinct vacuum configurations. The difference encodes both the 'linear and non-linear' or 'ordinary and null' memory effects $[26,27]$. If we could restrict ourselves to space-times for which $\left\{\stackrel{\circ}{D}^{-}\right\}=\left\{\stackrel{\circ}{D}^{+}\right\}$, then we could add this vacuum configuration to the universal structure at $\mathcal{I}$ and we would then have a reduction of the BMS group to the Poincaré group. But this would be too severe a restriction and we would be left with a very special class of isolated gravitating systems. Thus, if we wish to construct a framework that incorporates a sufficiently large class of physically interesting sources emitting gravitational waves, we have to live with the infinite 'vacuum degeneracy' and the corresponding enlargement of the Poincaré group to the infinite dimensional BMS group.

Remark: Our entire discussion involves $\mathcal{I}$ and is therefore formulated in terms of a conformal completion of the physical space-time. In terms of the physical space-time itself, the main point can be summarized as follows. Suppose we were to consider a class of space-times $\left(\hat{M}, \hat{g}_{a b}\right)$ which are such that we can extract a canonical Minkowski metric $\hat{\eta}_{a b}$ asymptotically and write $\hat{g}_{a b}=\hat{\eta}_{a b}+(1 / r) \hat{h}_{a b}$. Then we could use the Poincaré group selected by $\hat{\eta}_{a b}$ as our asymptotic symmetry group. However, if we allow generic sources of gravitational waves, then the presence of radiation in the asymptotic region prevents us from choosing a canonical $\hat{\eta}_{a b}$. Given one such $\hat{\eta}_{a b}$, we can perform a diffeomorphism corresponding to an angle-dependent translation and produce another $\hat{\eta}_{a b}^{\prime}$ which is equally good for our expansion. The Poincaré groups $\mathcal{P}$ and $\mathcal{P}^{\prime}$ selected by the two Minkowski metrics would then fail to agree even to leading order. Their translation subgroups would agree asymptotically, but the two sets of Lorentz groups will differ, and the difference will carry the imprint of the supertranslation relating $\hat{\eta}_{a b}$ and $\hat{\eta}_{a b}^{\prime}$. Returning to the language of null infinity, each Minkowski metric will enable us to select a 4-parameter family of cross-sections of $\mathcal{I}$ related by BMS translations. That is why the translation groups agree. However, the unprimed and the primed families of cross sections would be distinct, and the two families would be related by a supertranslation. Each family would determine a trivial connection in $\stackrel{\circ}{\Gamma}$ canonically, but the two 'vacua' $\{\stackrel{\circ}{D}\}$ and $\{\stackrel{\circ}{D}\}^{\prime}$ would be distinct and select distinct Poincaré subgroups $\mathcal{P}$ and $\mathcal{P}^{\prime}$ of the BMS group.

\section{Symplectic geometry of radiative modes}

So far, we have regarded $\mathcal{I}$ as the conformal boundary in the completed space-time $\left(M, g_{a b}\right)$ and used fields in $M$ to induce fields on $\mathcal{I}$. Now we wish to construct the phase space of all possible radiative modes of the gravitational field in full general relativity. Therefore we will now regard $\mathcal{I}$ as an abstract manifold, topologically $\mathbb{S}^{2} \times \mathbb{R}$, not attached to any physical space-time. It will be endowed with: i) the pairs $\left(q_{a b}, n^{a}\right)$, where $n^{a}$ is complete 
as in section II A (i.e., the universal structure); and, ii) connections $D$ that are compatible with the pairs $\left(q_{a b}, n^{a}\right)$ in the sense of section III A.

It is convenient to first fix a conformal frame $\left(q_{a b}, n^{a}\right)$ and, without any reference to a space-time, just compute the curvature $R_{a b c}{ }^{d}$ of any given $D$, extract $S_{a}{ }^{b}$, introduce the kinematical tensor $\rho_{a b}$, and define the news tensor $N_{a b}$ and the field ${ }^{\star} K^{a b}$ using equations displayed in section III A. These fields continue to satisfy all the relations at $\mathcal{I}$ we discussed even though $\mathcal{I}$ is not the boundary of any space-time. We can then introduce the equivalence relation (3.1) among connections $D$ and show that $N_{a b}$ and ${ }^{\star} K^{a b}$ depend only on the equivalence class. Finally, we can relate the structures associated with any two conformal frames $\left(q_{a b}, n^{a}\right)$ and $\left(q_{a b}^{\prime}=\omega^{2} q_{a b}, n^{\prime a}=\omega^{-1} n^{a}\right)$, by specifying the transformation property on the equivalence classes $\{D\}$ :

$$
\left(\left\{D_{a}\right\}^{\prime}-\left\{D_{a}\right\}\right) k_{b} \approx 2 \omega^{-1} k_{(b} D_{a)} \omega .
$$

where $\approx$ stands for equality modulo additive terms of the type $f q_{a b}$ for some $f$. We can then establish the transformation properties $N_{a b}^{\prime}=N_{a b}$ and ${ }^{\star} K^{\prime a b}=\omega^{-5 \star} K^{a b}$. Thus, all the structure that refers to the 'radiative modes' can be introduced directly on an abstractly defined $\mathcal{I}$ without reference to a physical space-time [23, 39, 44].

The phase space $\Gamma$ will consist of the connections $\{D\}$ subject to the condition that they approach some vacuum configurations $\left\{\stackrel{\circ}{D}^{\mp}\right\}$ at an 'appropriate rate' as one approaches the two 'ends', $i^{o}$ and $i^{+}$of $\mathcal{I}{ }^{3}$ This is the space of radiative modes at $\mathcal{I}$. To define the symplectic structure $\boldsymbol{\Omega}$ on $\boldsymbol{\Gamma}$, we first fix a conformal frame $\left(q_{a b}, n^{a}\right)$ on $\mathcal{I}$. Then, at any point $\{D\}$ of $\boldsymbol{\Gamma}$, the symplectic structure is a (weakly-non-degenerate) 2-form that assigns to any two tangent vectors $\sigma_{a b}, \sigma_{a b}^{\prime}$ at $\{D\}$ the following number:

$$
\left.\boldsymbol{\Omega}\right|_{\{D\}}\left(\sigma, \sigma^{\prime}\right):=\frac{1}{8 \pi G} \int_{\mathcal{I}}\left[\sigma_{a b} \mathcal{L}_{n} \sigma_{c d}^{\prime}-\sigma_{a b}^{\prime} \mathcal{L}_{n} \sigma_{c d}\right] q^{a c} q^{b d} \epsilon_{m n p} \mathrm{~d} S^{m n p} .
$$

Here $G$ is Newton's constant and $\epsilon_{a b c}$ is the 3 -form on $\mathcal{I}$ (unique up to sign) defined as follows: it is the inverse of the antisymmetric tensor field on $\mathcal{I}$ satisfying

$$
\epsilon^{m n p} \epsilon^{a b c} q_{m a} q_{n b}=n^{p} n^{c}
$$

(so that $\epsilon^{m n p} \epsilon_{m n p}=6$ ). Since $n^{a} \sigma_{a b}=0$, it follows that the right side of (3.14) is independent of the particular choice of the 'inverse' $q^{a b}$ of $q_{a b}$ made in this evaluation. Finally, under conformal rescalings $\left(q_{a b}^{\prime}=\omega^{2} q_{a b}, n^{\prime a}=\omega^{-1} n^{a}\right)$, one finds $\sigma_{a b}^{\prime}=\omega \sigma_{a b}, q^{\prime a b}=\omega^{-2} q^{a b}$ and $\epsilon_{a b c}^{\prime}=\omega^{3} \epsilon_{a b c}$, so that the right hand side of (3.14) remains unchanged. Hence the symplectic structure is insensitive to the initial choice of the conformal frame.

One can show that under the action of the BMS group, the symplectic structure is left invariant and one can therefore calculate the Hamiltonians generating these symplectomorphisms. This is a rather subtle and technically difficult calculation. However, the final result is rather simple [44]: the Hamiltonian corresponding to the BMS vector field $\xi^{a}$ is given by

$$
H_{\xi}(\{D\})=\frac{1}{16 \pi G} \int_{\mathcal{I}}\left[N_{a b}\left(\mathcal{L}_{\xi} D_{c}-D_{c} \mathcal{L}_{\xi}\right) \ell_{d}+2 N_{a b} \ell_{c} D_{d} \alpha\right] q^{a c} q^{b d} \epsilon_{m n p} \mathrm{~d} S^{m n p},
$$

\footnotetext{
${ }^{3}$ To make $\boldsymbol{\Gamma}$ into a proper symplectic space we need to specify function spaces more precisely. But that would require a long detour, and is beyond the scope of this brief report. One avenue, which involves $C^{\infty}$ fields (defined intrinsically on $\mathcal{I}$ ), is discussed in [44]. But there should be simpler options if one considers only $C^{k}$ fields in the spirit of the global existence results of the Christodoulou-Klainnerman type. This is an interesting open issue.
} 
where $\ell_{a}$ is any 1 -form on $\mathcal{I}$ satisfying $\ell_{a} n^{a}=-1$ and the function $\alpha$ is define via $\mathcal{L}_{\xi} q_{a b}=$ $2 \alpha q_{a b}$. The right side is independent of the particular choice of $\ell_{a}$, of the 'inverse' $q^{a b}$ of $q_{a b}$ and of the conformal frame $\left(q_{a b}, n^{a}\right)$ made in its evaluation. Mathematically, (3.16) provides a mapping from $\boldsymbol{\Gamma}$ to the dual of the BMS Lie algebra $\mathfrak{b}$; i.e., it is a momentum map associated with the natural action of the BMS group on the phase space $(\boldsymbol{\Gamma}, \boldsymbol{\Omega})$. Physically, $H_{\xi}$ represents the flux of the component of the 'BMS momentum' associated with the BMS generator $\xi^{a}$ across $\mathcal{I}$.

This momentum map has several interesting features. The salient among them can be summarized as follows.

i) Since the news tensor $N_{a b}$ vanishes at any vacuum configuration $\{\stackrel{\circ}{D}\}$, the flux of the entire BMS momentum across $\mathcal{I}$ vanishes identically in this case. This is just what one would expect of a 'classical vacuum'.

ii) If $\xi^{a}$ is a supertranslation, $\xi^{a}=f n^{a}$, then $\alpha=0$ and so the second term in the integrand vanishes and the first term simplifies to

$$
H_{\xi}(\{D\})=\frac{1}{16 \pi G} \int_{\mathcal{I}} N_{a b}\left[f S_{c d}+D_{c} D_{d} f\right] q^{a c} q^{b d} \epsilon_{m n p} \mathrm{~d} S^{m n p}
$$

In a Bondi frame, $S_{c d}=N_{c d}+\frac{1}{2} q_{c d}$ and hence

$$
H_{\xi}(\{D\})=\frac{1}{16 \pi G} \int_{\mathcal{I}} N_{a b}\left[f N_{c d}+f q_{c d}+D_{c} D_{d} f\right] q^{a c} q^{b d} \epsilon_{m n p} \mathrm{~d} S^{m n p} .
$$

iii) Recall that $D_{c} D_{d} f+f q_{c d}$ vanishes in a Bondi frame if and only if $f n^{a}$ is a BMS translation. So, in that case, the Hamiltonian simplifies further to

$$
H_{\xi}(\{D\})=\frac{1}{16 \pi G} \int_{\mathcal{I}}\left[f N_{a b} N_{c d}\right] q^{a c} q^{b d} \epsilon_{m n p} \mathrm{~d} S^{m n p}
$$

This is the celebrated expression of the flux of energy-momentum at null infinity that Bondi and Sachs had proposed in the 1960s. For a (future-directed) time translation, $f$ is positive and so the flux of energy is manifestly positive. They extracted this expression using the anticipated asymptotic behavior of the metric as one moves away from the sources in null directions, and the asymptotic field equations governing metric coefficients. It is this proposal that served to resolve the then controversial issue of whether gravitational waves are physical. As Bondi is said to have put it, they are real because they carry energy and we can heat water with them! In our discussion, the expression arises from principles of symplectic geometry, i.e., as the Hamiltonian generating BMS translations. This is a striking example of the rich interplay between geometry and physics that one encounters repeatedly in general relativity.

We will conclude with a discussion of an interesting subtlety that further illuminates the interplay between geometry and physics. In the early literature, it was thought that the flux of supermomentum is also given by Eq.(3.19), where now $f$ is a general function on the 2-sphere $S$ of generators of $\mathcal{I}$, rather than a BMS translation. But a careful analysis via symplectic geometry revealed that, for a general supertranslation, there is an extra term $D_{c} D_{d} f+2 f q_{c d}$, as in the integrand on the right side Eq. (3.18). In the older work, the phase space was constructed from 'shear tensors' rather than from connections and was thus regarded as a vector space rather than an affine space. This effectively amounts to fixing a $\{\stackrel{D}{ }\}$ once and for all as the origin and replacing every other $\{D\}$ with the tensor $\sigma_{a b}$ relating 
that $\{D\}$ to the fiducial $\{\stackrel{D}{D}\}$. However, as we discussed in section III C, supertranslations act non-trivially on vacuum configurations and therefore the fiducial $\{\stackrel{\circ}{D}\}$ is mapped to another $\left\{D^{\prime}\right\}$ under the action of $\mathcal{S} / \mathcal{T}$, invalidating the use of a fixed vector space structure in the older treatment. There are checks in terms of 'balance laws' that establish that the extra term arising from the shift of the origin is essential $[38,44]$. Thus, the fact that the phase space has the structure of an affine space rather than a vector space has direct physical consequences.

Finally the phase space $(\boldsymbol{\Gamma}, \boldsymbol{\Omega})$ and the subsequent constructions of the Hamiltonians are completely parallel to those used in the analysis of Maxwell and Yang-Mills fields in Minkowski space-time at $\mathcal{I}$; the only difference is that the symmetry group is simply the Poincaré group rather than the BMS. In these examples, if there are no sources (i.e., if we have 'pure' gauge fields), one can show that the phase space of radiative modes is isomorphic with the 'covariant phase space' constructed from the space of solutions [44]. The same result has been obtained for source-free solutions to Einstein's equations [45] but without a rigorous treatment of function spaces. In light of results on global existence for 'small' data [12, 14] that have been established since then, it should be possible to put results of [45] on a sounder mathematical footing. This extension would be of considerable interest both for classical and quantum gravity.

\section{QUANTUM ASPECTS}

Null infinity is especially well-suited to discuss the $S$-matrix theory of zero rest mass fields. In the case of the Maxwell field in Minkowski space-time, one can construct the Fock space of quantum states directly from the phase space of radiative modes at $\mathcal{I}^{\mp}$, without reference to the interior of space-time. They serve as the 'in' and 'out' photon states for the $S$-matrix. Furthermore, the description at $\mathcal{I}$ provides a clean, geometric understanding of the subtle infra-red problems [46] one encounters in the scattering theory in quantum electrodynamics [23].

In the gravitational case, the situation is more complicated, first because of the absence of a Minkowski space-time in the background, and second because the gravitational field acts as its own source. The $\mathcal{I}$-framework enables one to surmount the first difficulty in that one can construct the asymptotic Hilbert spaces of states on $\mathcal{I}^{\mp}$ for full non-linear general relativity. Currently significant advances are being made in calculating the $S$-matrix from the Hilbert space at $\mathcal{I}^{-}$to that at $\mathcal{I}^{+}$using twistor methods (see, e.g., [30, 31]). Furthermore, using the BMS group one can assign mass and spin to these asymptotic quanta and show that they have $m=0$ and $s=2$. Thus, even in full general relativity the elementary quanta can be identified with gravitons at null infinity. Finally, one can show that there is a direct correspondence between the 'vacuum degeneracy' discussed in section III B and infrared issues in the quantum theory associated with 'soft gravitons'. However, these issues are much more complicated than in the Maxwell case because of the second difficulty the 'non-Abelian' character of general relativity [23] - and constitute a subject of ongoing investigations [32-34].

I will now provide a brief overview of all these quantum aspects. This discussion will include only those issues that are likely to interest the geometric analysis community and will therefore not cover recent results, nor will the treatment of topics covered be comprehensive. 


\section{A. Quantization of the radiative modes}

The phase space $(\boldsymbol{\Gamma}, \boldsymbol{\Omega})$ of radiative modes provides a natural point of departure for quantization. In field theories, because of the presence of an infinite number of degrees of freedom, von-Neumann's [47] celebrated uniqueness theorem (on representations of the canonical commutation relations) is no longer applicable. So we are led to an algebraic approach where one first constructs the appropriate algebra of observables and then seeks its representations on Hilbert spaces. In the case when the phase space is linear - as, e.g., in the Maxwell theory - the 'elementary' observables generating these algebras are taken to be linear functions on phase space. Their Hamiltonian vector fields are constant on the phase space. Our phase space $\Gamma$ is an affine space and we can again seek functions whose Hamiltonian vector fields are constant. It turns out that these can be obtained by smearing the news tensor $N_{a b}$ by test fields. Let $f_{a b}$ denote symmetric second rank test fields on $\mathcal{I}$, transverse to $n^{a}$, that belong to the Schwarz space $\mathcal{S}$ of fields which, together with all their derivatives decrease rapidly (as one moves to $i^{o}$ and $i^{ \pm}$along $\mathcal{I}^{ \pm}$). Then the 'elementary' classical observables on $\Gamma$ turn out to be

$$
[N[f]](\{D\}):=-\frac{1}{8 \pi G} \int_{\mathcal{I}} N_{a b} f_{c d} q^{a c} q^{b d} \epsilon_{m n p} d S^{m n p} .
$$

The Hamiltonian vector field $X_{N[f]}$ is just the constant vector field on $\boldsymbol{\Gamma}$, represented by $f_{a b}$. The Poisson brackets are given by

$$
\left\{N[f], N\left[f^{\prime}\right]\right\}_{\mathrm{PB}}=-\frac{1}{8 \pi G} \int_{\mathcal{I}}\left[f_{a b} \mathcal{L}_{n} f_{c d}^{\prime}-f_{a b}^{\prime} \mathcal{L}_{n} f_{c d}\right] q^{a c} q^{b d} \epsilon_{m n p} d S^{m n p}=\Omega\left(f, f^{\prime}\right) .
$$

Note that the right side is a constant. The algebra $\mathfrak{A}$ of quantum operators is generated by the (abstractly defined) $\hat{N}[f]$ 's. More precisely, $\mathfrak{A}$ is the free $\star$-star algebra generated by the $\hat{N}[f]$ subject to the relations

$$
\hat{N}[f]+\lambda \hat{N}\left[f^{\prime}\right]=\hat{N}\left[f+\lambda f^{\prime}\right] ; \quad \hat{N}^{\star}[f]=\hat{N}[f] ; \quad \text { and } \quad\left[\hat{N}[f], \hat{N}\left[f^{\prime}\right]\right]=i \hbar \Omega\left(f, f^{\prime}\right) \mathbb{I}
$$

where $\lambda \in \mathbb{R}$ and $\mathbb{I}$ is the identity operator. In finding representations, it is more convenient to work with the Weyl Algebra $\mathfrak{W}$, generated by $\hat{W}[f]=\exp [(i / \hbar) \hat{N}[f])$ because the vector space generated by $W[f]$ is closed under the product:

$$
\hat{W}[f] \hat{W}\left[f^{\prime}\right]=e^{-\frac{i}{2 \hbar} \boldsymbol{\Omega}\left(f, f^{\prime}\right)} \hat{W}\left[f+f^{\prime}\right] .
$$

To find its representations, one can use a standard construction due to Gel'fand, Naimark and Segal (GNS) [48]: Given a positive linear functional on W', the GNS construction yields a canonical, cyclic representation of $\mathfrak{W}$ on a Hilbert space $\mathcal{H}$. The cyclic state in $\mathcal{H}$ is called 'the vacuum' (and denoted $|0\rangle$ ) because any other state in the Hilbert space can be obtained by acting repeatedly by elements of $\mathfrak{W}$ on it. The positive linear functional on $\mathfrak{W}$ is called the vacuum expectation value function (or VEV) because in the GNS representation of $\mathfrak{W}$ on $\mathcal{H}$, it yields precisely the expectation values $\langle 0|\hat{W}[f]| 0\rangle$ of $\hat{W}[f]$ in the state $|0\rangle$.

To select the VEV, it is simplest to introduce a Kähler structure on the space $\mathcal{S}$ of test fields. By mimicking the procedure used for Maxwell fields in Minkowski space-time, one is led to first introduce a complex structure $J$ by decomposing the fields $f_{a b}$ into positive 
and negative frequency parts, using the affine parameter $u$ on the integral curves of $n^{a}$ : $J \cdot f_{a b}=i f_{a b}^{+}-i f_{a b}^{-}$with

$$
f_{a b}^{+}(u, \theta, \phi)=\int_{o}^{\infty} \tilde{f}_{a b}(\omega, \theta, \phi) e^{-i \omega u} \mathrm{~d} \omega \quad \text { and } \quad f_{a b}^{-}=\left(f_{a b}^{+}\right)^{\star},
$$

where $u, \theta, \phi$ are the obvious coordinates on $\mathcal{I}$. One can readily verify that: i) $J$ does not depend on the particular conformal frame chosen (i.e., $n^{a}$ ) or the coordinates used in its construction; and, ii) it is compatible with the symplectic structure $\Omega$. Thus, we have a Kähler space. Denote by $\mathfrak{h}$ the Cauchy completion of $\mathcal{S}$ with respect to the resulting Hermitian inner product

$$
\left\langle f \mid f^{\prime}\right\rangle:=\frac{1}{2}\left(\Omega\left(f, J f^{\prime}\right)+i \Omega\left(f, f^{\prime}\right)\right) .
$$

This Hilbert space will feature prominently in what follows.

We can now define the required VEV on $\mathfrak{W}$ :

$$
\langle 0|\hat{W}[f]| 0\rangle:=e^{-\frac{1}{2 \hbar} \boldsymbol{\Omega}(f, J f)}
$$

and carry out the GNS construction. The underlying representation space $\mathcal{H}$ is naturally isomorphic to the symmetric Fock space based on $\mathfrak{h}$ :

$$
\mathcal{H}=\oplus_{n=0}^{\infty} \mathfrak{h}_{n}^{\text {sym }}
$$

where for $n>0$, the Hilbert space $\mathfrak{h}_{n}^{\text {sym }}$ is the symmetric tensor product of $n$ copies of $\mathfrak{h}$ and $\mathfrak{h}_{0}^{\text {sym }}=\mathbb{C}$. The normalized vector in $\mathbb{C}$ is the 'vacuum', and elements of $\mathfrak{h}_{n}^{\text {sym }}$ are obtained by repeated action of creation operators $a^{\dagger}[f]=(1 / 2)(N[f]+i N[J f])$ on the vacuum. This representation of the Weyl algebra of news operators provides the asymptotic Hilbert spaces at $\mathcal{I}$ for the $S$-matrix theory. Note that the underlying phase space $(\boldsymbol{\Gamma}, \boldsymbol{\Omega})$ played a key role both in the construction of the news operators and in the introduction of the Kahler structure that lies at the heart of the representation.

\section{B. Properties of the representation and infrared issues}

Physically, it is convenient to tie this representation to the classical phase space in order to make the correspondence between the classical and quantum theories transparent. For definiteness, let us fix a fiducial classical vacuum $\{\stackrel{\circ}{D}\}_{0}$. One can interpret the quantum vacuum state $|0\rangle$ as the coherent state in $\mathcal{H}$, peaked at this configuration. Recall that any two connections in the phase space $\boldsymbol{\Gamma}$ are related by a symmetric, second rank, transverse tensor field $\sigma_{a b}$. Consider the subspace $\boldsymbol{\Gamma}_{0}$ of $\boldsymbol{\Gamma}$ consisting of connections $\{D\}$ which are related to the given $\{\stackrel{\circ}{D}\}_{0}$ by a $\sigma_{a b}$ which has finite norm with respect to (4.6). We can regard $\boldsymbol{\Gamma}_{0}$ as a vector space with $\{\stackrel{\circ}{D}\}_{0}$ as its origin and label every element $\{D\}$ of $\boldsymbol{\Gamma}_{0}$ with the corresponding tensor field $\sigma_{a b}$. Then, there is a natural isomorphism, $\sigma_{a b} \rightarrow f_{a b}=\sigma_{a b}$, between $\boldsymbol{\Gamma}_{0}$ and the 1-particle Hilbert space $\mathfrak{h}$. Recall that, in any Fock space, each 1-particle state defines a coherent state. The coherent state $\Psi_{f}$ is then the 'canonical' semi-classical quantum state corresponding to the classical phase space point $\{D\}$ labeled by $\sigma_{a b}=f_{a b}$. Note that because of the requirement of finiteness of norm, $\boldsymbol{\Gamma}_{0}$ does not contain any classical 
vacuum $\{\stackrel{\circ}{D}\}$ other than $\{\stackrel{\circ}{D}\}_{0}$. Therefore, our Hilbert space does not have any 1-particle states or coherent states corresponding to the other classical vacua.

Recall from section III B that each vacuum state selects a Poincaré subgroup $\mathcal{P}$ of $\mathfrak{B}$. It is easy to check that the subgroup selected by $\{\stackrel{\circ}{D}\}_{0}$ preserves the 1-particle Hilbert space $\mathfrak{h}$. Furthermore, this action provides a unitary, representation of that Poincaré group. One can show that it can be decomposed into two irreducible representations. Every irreducible representation of $\mathcal{P}$ is labeled by the eigenvalues of its Casimir operators, mass and spin. An explicit calculation shows that the eigenvalue of the mass operator is 0 on both irreducible representations and those of the spin operator are \pm 2 . When $m=0$, the spin vector is either parallel or anti-parallel to the 4-momentum and the resulting configurations are said to have positive and negative helicity respectively. In explicit terms, $f_{a b} \in \mathfrak{h}$ has positive/negative helicity if its positive frequency part is 'self-dual/anti self-dual' in the sense that $\epsilon^{m n p} \ell_{p} q_{n b} f_{a m}^{+}=\mp i f_{a b}^{+}$for any $\ell_{a}$ on $\mathcal{I}$ satisfying $\ell_{a} n^{a}=-1$. (For details, see [23], and for the simpler Maxwell example, [49]).

The classical expressions of the fluxes $H_{\xi}$ of BMS momenta (3.16) can be promoted to self-adjoint quantum operators $\hat{H}_{\xi}$ on $\mathcal{H}$. These operators provide us with the notion of fluxes of the BMS momenta in the quantum theory. It turns out that the Kähler structure selected by the positive and negative frequency decomposition guarantees that the answers one would get in the quantum theory are fully compatible with those in the classical theory. More precisely, if $\left|\Psi_{\{D\}}\right\rangle$ is the coherent state in $\mathcal{H}$ peaked at a point $\{D\}$ in $\boldsymbol{\Gamma}_{0}$, then

$$
\left\langle\Psi_{\{D\}}\left|\hat{H}_{\xi}\right| \Psi_{\{D\}}\right\rangle=H_{\xi}(\{D\}) .
$$

Furthermore, this physical condition suffices to determine the Kähler structure used in our quantization uniquely. These results pave the way to establish that there exists a welldefined $S$-matrix theory on the sector of the asymptotic Hilbert space $\mathcal{H}$ that is spanned by coherent states peaked at the classical configurations $\{D\} \in \boldsymbol{\Gamma}_{0}$ that are also sufficiently close to $\{D\}_{0}$ in the sense that they are induced on $\mathcal{I}^{ \pm}$by the evolution of 'small initial data' à la $[12,14]$.

If the data is not 'small', the evolution may lead to the formation of a black hole in classical general relativity. In this case, $\mathcal{I}^{+}$would not serve as a good future boundary for the $S$-matrix theory. Furthermore, this can happen even when the incoming gravitons at $\mathcal{I}^{-}$ have completely 'tame' frequencies. Therefore one would expect the $S$-matrix description to be inadequate in these circumstances, even though they occur well away from the Planck regime. This limitation was realized rather soon after the introduction of the asymptotic quantization program and drained the motivation from making a serious attempt to construct the quantum $S$-matrix at that time. Since then, advances in geometric analysis have furnished detailed results on the class of initial data that do lead to a well-defined $S$-matrix on the classical side, and twistor methods are now enabling concrete progress on the quantum side. By combining these results, one may be able to obtain sharp results on the class of asymptotic states in $\mathcal{H}$ for which the quantum scattering operator is well defined to leading order in perturbation theory. This would resolve the 30 year old tension between black hole formation in the classical theory and the perturbative $S$-matrix program in the quantum theory.

Finally, the geometric structure at $\mathcal{I}$ also sheds new light on the infrared issues associated with gravity. Consider any point $\{D\}$ in $\boldsymbol{\Gamma}_{0}$ labeled by the field $\sigma_{a b}$. One can show that the news tensor $N_{a b}$ of $\{D\}$ is given by $N_{a b}=2 \mathcal{L}_{n} \sigma_{a b}$. But recall that for $\sigma_{a b}$ to define an element of $\mathfrak{h}$ (i.e., a 1-graviton state), its norm under (4.6) must be finite. This turns out to 
be a strong requirement, for it implies

$$
Q(\theta, \phi):=\int_{-\infty}^{\infty} \mathrm{d} u N_{a b}(u, \theta, \phi)=0 .
$$

If $Q(\theta, \phi) \neq 0$, the norm (4.6) is infrared divergent (i.e. diverges because the integral in (4.5) extends to $\omega=0)$. Hence $Q(\theta, \phi)$ is referred to as the 'infrared charge' of $\{D\}$. Note that mathematically (4.10) amounts to an infinite number of conditions because the integral over $u$ has to vanish for each generator of $\mathcal{I}$, labeled by $\theta, \phi$. To understand its physical content, it is best to recast it in terms of connections. It is straightforward to show that (4.10) is equivalent to asking that, for a connection $\{D\}$ to be in $\boldsymbol{\Gamma}_{0}$, it must approach the same vacuum configuration at both ends of $\mathcal{I}$. (On $\mathcal{I}^{+}$the two ends are $i^{o}$ and $i^{+}$and on $\mathcal{I}^{-}$they are $i^{-}$and $i^{o}$.) As we discussed in section III B, because of the memory effect [26], this is physically too stringent a requirement. In scattering processes one does not expect it to be satisfied except in rather exceptional circumstances.

This means that, physically, it would be unreasonable to restrict oneself to the Fock representation we have constructed above. However, it is relatively straightforward to enlarge the asymptotic Hilbert spaces by allowing suitable 'displaced Fock representations' [23]. This enlargement is necessary already in quantum electrodynamics where, even after renormalization, we do not have a well-defined $S$-matrix order by order in perturbation theory because of the infrared problems associated with photons which are completely analogous when formulated in terms of $\mathcal{I}$. In that case, one can find the 'minimal enlargement' to make the $S$-matrix well-defined in an efficient and elegant manner $[23,50]$. However, the procedure makes crucial use of the fact that the photons themselves do not act as sources of the quantum Maxwell field; the theory is an Abelian gauge theory. Although this simplification is not available in gravity, there have been interesting recent advances within perturbation theory [51]. It would be interesting to investigate if these results can lead to a coherent quantum theory of the 'small data' sector for which we have global existence theorems in the classical theory.

\section{DISCUSSION}

Structure of null infinity is both subtle and rich. In the classical theory, it has led to some surprising results. In particular, there is a 'vacuum degeneracy' in the phase space of connections directly related to the presence of supertranslations, i.e., to the fact the asymptotic symmetry group is the infinite dimensional BMS group $\mathfrak{B}$ rather than the 10 dimensional Poincaré group. The BMS group acts via symplectomorphisms on the phase space and their Hamiltonians provide us with the expressions of the BMS momenta across $\mathcal{I}$ that are important for the physics of gravitational waves. This review focused on the geometric aspects of null infinity, rather than on global issues on the existence and uniqueness of solutions to Einstein's equations. A summary of the rich set of results in that area can be found, e.g., in $[12-14,16,17]$.

In the quantum regime, the structure at $\mathcal{I}$ enables one to construct suitable operator algebras and their representations. The Hilbert space underlying these representations provides the asymptotic states for the $S$-matrix theory. This framework provides a precise sense in which gravitons arise in full non-linear gravity, without having to make expansions around Minkowski space-time. Furthermore, since one can introduce abstract boundaries 
$\mathcal{I}^{ \pm}$equipped with structures that are sufficiently rich to carry out these constructions without reference to the space-time interior, one can now envisage various space-time geometries as paths, or histories, that interpolate between the fixed, well-defined asymptotic states on $\mathcal{I}^{ \pm}$. Therefore, there is also a potential for calculating a non-perturbative sum over histories, e.g., along the lines of spin foams (see, e.g., [52]), or, by allowing classical geometries with different topologies in the bulk in the spirit of Wheeler's 'space-time foam' paradigm.

In both regimes interesting open issues still remain at the interface of geometric analysis and gravitational physics. Perhaps the most surprising among these is a set related to the cosmological constant $\Lambda$. Every issue discussed in this overview assumes $\Lambda=0$, while by now we have very strong indications from observations that $\Lambda$ is small but positive. At first, one might expect that it would be trivial to extend the interplay between geometry and physics to accommodate the presence of a non-zero $\Lambda$. And indeed, there have been a number of interesting results for the $\Lambda<0$ case. For $\Lambda>0$, a global existence result, due to Friedrich [53], has been available for a long time. However, from a physical perspective, very little is known in the $\Lambda>0$ case. As of now, we do not have a physically useful positive energy theorem because, for $\Lambda>0$, the asymptotic symmetry representing 'time translation' that defines energy is space-like near $\mathcal{I}$. We also do not have the analog of the Bondi news. So, we do not even have a gauge invariant characterization of gravitational waves in full general relativity, let alone the phase space of radiative modes and formulas for Hamiltonians representing fluxes of energy-momentum! Thus, even a tiny cosmological cosmological constant casts a long shadow, if it is positive [42], as in the real universe. Extensions of the rich results outlined in this overview to the $\Lambda>0$ case represent a vast field of opportunities for new, interesting results for both physics and mathematics communities. I hope that this overview will help enhance the interaction between the two.

\section{Acknowledgments}

This work was supported in part by the NSF grants PHY-1205388, PHY-1505411 and the Eberly research funds of Penn State. I thank Béatrice Bonga and Aruna Kesavan for a careful reading of the manuscript and the referee for useful suggestions.

[1] R. Arnowitt, S. Deser and C. W. Misner, The dynamics of general relativity, In: Gravitation: An Introduction to Current Research, edited by L. Witten (Wiley \& Sons, New York, 1962).

[2] A. Ashtekar and R. O. Hansen, A Unified Treatment of Spatial and Null Infinity in General Relativity: Universal Structure, Asymptotic Symmetries and conserved Quantities at Spatial Infinity, J. Math. Phys. 19, 1542-1566 (1978).

[3] A. Ashtekar, Asymptotic Structure of the Gravitational Field at Spatial Infinity, In: General Relativity and Gravitation: One Hundred Years After the birth of Albert Einstein, edited by A. Held, (Plenum, New York, 1980).

[4] R. Schoen and S. T. Yau, On the proof of the positive mass conjecture in general relativity, Commun. Math. Phys. 65, 45 (1979);

Proof of the positive mass theorem. II, Commun. Math. Phys. 79, 231 (1981).

[5] E. Witten, A new proof of the positive energy theorem, Commun. Math. Phys. 80, 381 (1981). 
[6] D. Kenneflick, Who is afraid of the referee? Einstein and gravitational waves, Talk at Stony Brook, October 2005, http://dafix.uark.edu/ danielk/Physics/gravwave.html

[7] H. Bondi, Radiation from an isolated system, In: Proceedings on theory of gravitation, edited by I. Infeld (Guthier Villars, Paris and PWN Editions Scientific de Pologne, Warszawa (1964)).

[8] R. Sachs, Asymptotic symmetries in gravitation theory, Phys. Rev. D128, 2851-2864 (1962).

[9] E. T. Newman and K. P. Tod, Asymptotically flat space-times, In: General Relativity and Gravitation: One Hundred Years After the birth of Albert Einstein, edited by A. Held, (Plenum, New York, 1980).

[10] R. Penrose, Zero rest mass fields including gravitation: asymptotic behavior, Proc. R. Soc. (London) A284, 157-203 (1965);

R. Penrose and E. T. Newman, New conservation laws for zero rest mass fields in asymptotically flat space-times, Proc. R. Soc. (London) A305, 175-204 (1968).

[11] G. J. Gallaway, P. Miao and R. Schoen, Initial data and Einstein constraint equations, in In: General Relativity and Gravitation: A Centennial perspective, Edited by A. Ashtekar, B.K. Berger, J. Isenberg, M.A.H. MacCallum (CUP, Cambridge, 2015)

[12] D. Christodoulou and S. Klainnerman, The global non-linear stability of Minkowski space (PUP, Princeton, 1993).

[13] H. Friedrich, Conformal Einstein evolution. In: The Conformal Structure of Spacetime: Geometry, Analysis, Numerics, Edited by J. Frauendiener, H. Friedrich, (Springer, Berlin, 2002).

[14] L. Bieri, An Extension of the stability theorem of the Minkowski space in general relativity, Journal of Differential Geometry. 86. no.1. (2010). 17-70.

L. Bieri, Extensions of the stability theorem of the Minkowski space in general relativity. Solutions of the Einstein vacuum equations. (AMS, International Press, 2009).

[15] N. Zipser, Extensions of the stability theorem of the Minkowski space in general relativity. Solutions of the Einstein-Maxwell equations. (AMS, International Press, 2009).

[16] H. Friedrich, Geometric asymptotics and beyond, arXiv:1411.3854.

[17] S. Aretaki and I. Rodnianski, The Cauchy problem in general relativity, In the volume cited in Ref. 11.

[18] L. Blanchet, Gravitational radiation from post-Newtonian sources and inspiralling compact binaries, Living Rev. Relativity, 9, 4 (2006).

[19] M. Choptuik, L. Lehner and F. Pretorius, Probing strong field gravity through numerical simulations, In the volume cited in Ref. 11.

[20] Les Astre Occlus, edited by B. DeWitt and C. M. DeWitt (Gordon and Breach, New York, 1973).

[21] A. Ashtekar, V. Taveras and M. Varadarajan, Information is not lost in the evaporation of 2-dimensional black holes, Phys. Rev. Lett. 100, 211302 (2008).

[22] A. Ashtekar, F. Pretorius and F. Ramazanoglu, Surprises in the evaporation of two dimensional black holes, Phys. Rev. Lett. 106, 161303 (2011)

Evaporation of two dimensional black holes, Phys. Rev. D83, 044040 (2011).

[23] A. Ashtekar, Asymptotic quantization of the gravitational field, Phys. Rev. Lett. 46, 573-577 (1981);

A. Ashtekar, Quantization of the radiative modes of the gravitational field, In: Quantum Gravity 2; Edited by C. J. Isham, R. Penrose, and D. W. Sciama (Oxford University Press, Oxford, 1981).

[24] A. Ashtekar, Asymptotic Quantization (Bibliopolis, Naples (1987)). 
[25] D. C. Christodoulou, Nonlinear nature of gravitation and gravitational-wave experiments, Phys. Rev. Lett. 67, 1486-1489 (1991).

[26] J. Frauendiener, Note on the memory effect, Class. Quantum Grav. 9, 1639-1641 (1992).

[27] L. Bieri and D. Garfinkle, A perturbative and gauge invariant treatment of gravitational wave memory, Phys. Rev. D89, 084039 (2014).

[28] P. J. McCarthy, Real and complex asymptotic symmetries in quantum gravity, irreducible representations, polygons, polyhedra, and the A, D and E series, Phil. Tras. R. Soc. London A338, 271-299 (1992).

[29] P. J. McCarthy and E. Melas, On the irreducible representations of the ultrahyperolic BMS group, Nucl. Phys. B653, 369-399 (2003).

[30] T. Adamo, E. Casali and D. Skinner, Ambitwistor strings and the scattering equations at one loop, JHEP 1404, 104 (2014).

[31] N. Arkani-Hamed and J. Trnka, The Amplituhedron, arXiv:1312.2007.

[32] T. He, V. Lysov, P. Mitra, and A. Strominger, BMS supertranslations and Weinberg's soft graviton theorem, arXiv: 1401.7026.

[33] T. Adamo, E. Casali and D. Skinner, Perturbative gravity at null infinity, arXiv:1405.5122

[34] M. Campiglia and A. Laddha, On asymptotic symmetries and subleading soft graviton theorem, arXiv: 1408.2228

[35] R. Penrose and W. Rindler, Spinors and Space-time, Vol. 1 and 2 (CUP, Cambridge 1987).

[36] R. Geroch and G. T. Horowitz, Asymptotically Simple Does Not Imply Asymptotically Minkowskian, Phys. Rev. Lett. 40, 203-206 (1978).

[37] A. Ashtekar and B. G. Schmidt, Null infinity and Killing fields, J. Math. Phys. 21, 862-867 (1980).

[38] W. T. Shaw, Symplectic geometry of null infinity and two-surface twistors, Class. Quant. Grav. 1, L33-L37 (1984);

T. Dray, Angular momentum at null infinity, Class. Quant. Grav. 1, 15-26 (1984).

[39] A. Ashtekar, Radiative degrees of freedom of the gravitational field in exact general relativity, J. Math. Phys. 22, 2885-2895 (1981).

[40] J. Kánnár, on the existence of $C^{\infty}$ solutions to the asymptotic characteristic initial value problem in general relativity, Proc. R. Soc. London A452, 945-952 (1996).

[41] R. Geroch, In: Asymptotic structure of space-time, Edited by L. Witten, pp1-106 (Plenum, New York,1976).

[42] A. Ashtekar, B. Bonga and A. Kesavan, Asymptotics with a positive cosmological constant: I. Basic framework, Class. Quant. Grav. 32, 025004 (2014).

[43] E. T. Newman and R. Penrose, Note on the Bondi Metzner Sachs group, J. Math. Phys. 7 863-870 (1965).

[44] A. Ashtekar and M. Streubel, Symplectic geometry of radiative modes and conserved quantities at null infinity, Proc. R. Soc. (London) A376, 585-607 (1981).

[45] A. Ashtekar and A. Magnon, On the symplectic structure of general relativity, Comm. Math. Phys. 86, 55-68 (1982).

[46] P. P. Kulish and L. D. Faddeev, Asymptotic conditions and infrared divergences in quantum electrodynamics, Teor. Mat. Fiz. 4 153-170 (1970).

[47] J. von Neumann, Die Eindeutigkeit der Schrödingerschen Operatoren, Math. Ann., $104570-$ 578 (1931).

[48] I. M. Gel'fand and M. A. Naimark, On the embedding of normed rings into the ring of operators in Hilbert space, Mat. Sobrn. 12, [54] 197-217 (1943); 
I. E. Segal, Postulates of general quantum mechanics, Ann. Math. 48 930-948 (1947).

[49] A. Ashtekar, A note on self-duality and helicity, J. math. Phys. 27, 824-827 (1986).

[50] A. Ashtekar and K. S. Narain, Infrared problems in quantum field theory and Penrose's null infinity, Syracuse University Report, Presented at the VIth International Conference on Mathematical Physics (1981).

[51] J. Ware, R. Saotome and R. Akhouri, construction of an asymptotic S-matrix for perturbative gravity, JHEP 10, 159 (2013)

[52] A. Ashtekar, M. Reuter and C. Rovelli, From general relativity to quantum gravity, In the volume cited in Ref. 11.

[53] H. Friedrich, On the global existence and the asymptotic behavior of solutions to the EinsteinMaxwell-Yang-Mills equations, J. Diff. Geo. 34, 275-345 (1991). 